\title{
Concepts for Domain Wall Motion in Nanoscale Ferromagnetic Elements due to Spin Torque and in Particular Oersted Fields
}

\author{
Mathias Kläui $^{1 *}$, Dennis Ilgaz ${ }^{1}$, Lutz Heyne ${ }^{1}$, June-Seo Kim$^{1}$, Olivier Boulle ${ }^{1}$, Christine Schieback $^{1}$, \\ Fabian Zinser', Stephen Krzyk ${ }^{1}$, Mikhail Fonin ${ }^{1}$, Ulrich Rüdiger ${ }^{1}$, Dirk Backes ${ }^{1,2}$, \\ Laura J. Heyderman ${ }^{2}$, T. O. Mentes ${ }^{3}$, and A. Locatelli ${ }^{3}$ \\ ${ }^{1}$ Fachbereich Physik, Universität Konstanz, Universitätsstr. 10, D-78457 Konstanz, Germany \\ ${ }^{2}$ Laboratory for Micro- and Nanotechnology, Paul Scherrer Institut, CH-5232 Villigen PSI, Switzerland \\ ${ }^{3}$ Sincrotrone Trieste S.C.p.A., 34012 Basovizza, Trieste, Italy
}

(Received 21 November 2008, Received in final form 18 December 2008, Accepted 11 February 2009)

\begin{abstract}
Herein, different concepts for domain wall propagation based on currents and fields that could potentially be used in magnetic data storage devices based on domains and domain walls are reviewed. By direct imaging, we show that vortex and transverse walls can be displaced using currents due to the spin transfer torque effect. For the case of field-induced wall motion, particular attention is paid to the influence of localized fields and local heating on the depinning and propagation of domain walls. Using an Au nanowire adjacent to a permalloy structure with a domain wall, the depinning field of the wall, when current pulses are injected into the Au nanowire, was studied. The current pulse drastically modified the depinning field, which depended on the interplay between the externally applied field direction and polarity of the current, leading subsequently to an Oersted field and heating of the permalloy at the interface with the Au wire. Placing the domain wall at various distances from the Au wire and studying different wall propagation directions, the range of Joule heating and Oersted field was determined; both effects could be separated. Approaches beyond conventional field- and current-induced wall displacement are briefly discussed.
\end{abstract}

Keywords : domain wall motion, oersted field induced domain wall dynamics, magnetic shift register, joule heating, spin torque

\section{Introduction}

In the past, magnetic nanostructures have been at the heart of a multitude of devices ranging from sensing applications to data storage. Probably the best known storage device is the magnetic disc drive [1], pioneered in the 1950s by IBM with the RAMAC. Since then, storage density has seen a gigantic exponential increase. While hard drives continue to excel in the high capacity market, they nonetheless entail disadvantages that have led to other memory concepts replacing them for applications, such as lower density mobile storage. One of the key problems is the mechanical motion of the media that poses reliability questions and can lead to catastrophic

*Corresponding author: Tel: +49-7531-883786,

Fax:+49-7531-883789, e-mail: mathias.klaeui@uni-konstanz.de; also at: Zukunftskolleg, Universität Konstanz, 78457 Konstanz, Germany. failure in the case of mechanical shock. Another successful form of magnetic memory is magnetic tape, with a huge capacity but obvious limitations when it comes to random access [2]. Like the disc drive, tape drives physically move the media (tape), leading to wear. Another disadvantage that physical motion brings is power consumption, which in laptops leads to a significant portion of the power being used by the hard drive. For many of the growing memory markets, in particular in mobile applications (laptops, PDAs), low power combined with a solid-state technology is required. Different approaches have been suggested based on a range of technologies. For this review, the focus will lie on magnetic materials and thus, for magnetic data storage, a paradigm shift away from hard drives and tape is required. One exciting approach recently proposed is based on magnetic nanowires with magnetic domains acting as the bits (Fig. 1). The interface between 2 domains where magnetization points in opposite directions is called a magnetic domain wall, in which the 




Fig. 1. (colour online): Schematic of a magnetic wire with domains. The colour and the arrows indicate the magnetization direction. The domains with the magnetization pointing to the right correspond to a logical 0 and magnetization pointing to the left corresponds to a 1 (in this example the digital information 010011 is encoded).

spins turn by $180^{\circ}$ and the nanoscale spin structure depends on the material and geometry. At first sight, the design looks similar to tape, but rather than shifting the media with the data as in the case of tape, here the data is shifted within the media that stays physically fixed, leading to much faster possible access times and in particular, eliminating all mechanical motion. Moreover, the device only comprises 1 write or read element for potentially tens to hundreds of bits, which can make it cost effective. Furthermore, this simplifies integration with the necessary semiconductor electronics that can be more compact and thus lead to higher storage densities compared to magnetic random access memory [3]. Prominent suggestions for concrete realizations of such a device are the racetrack device envisaged by S. S. P. Parkin $[4,5]$ and the shift register proposed by R. Cowburn [6]. To make such a device useful for memory storage, 3 key tasks have to be performed, the data needs to be written, read, and selected (meaning that the bit to be read or written needs to be moved to the read or write element). The writing can be performed by using appropriately designed strip lines that generate fields to reverse the magnetization and thus "write" a bit. This can be compared to the well-established writing of bits in magnetic hard drives. The reading relies on the sensing of the domains or domain walls, very similar to hard drives, so that the well-established magnetoresistive sensing, based on giant magnetoresistance read heads $[1,7]$ or tunneling magnetoresistance read heads [7], can be employed. However, it is the domain selection by motion of the domains and domain walls that is novel and requires alternative approaches. Thus, the dynamic behaviour of geometrically-confined domain walls (DWs) and their motion due to applied fields and induced current pulses have become a topic of growing interest in the last years due to the intricate magnetic properties present in geometrically-confined ferromagnetic structures [8]. In this review, current-induced wall displacement will first be addressed, followed by a focus on displacement by fields, and finally a brief mention of further schemes still in earlier stages of development. In addition to data storage, sensing magnetic fields by domain wall displacement has been suggested [9] and domain walls have most prominently been proposed for logic. Such logic based on domain walls has been extensively researched and the necessary logic functions such as NOT, AND, and other gates have been developed [10,11].

\section{Current-induced Domain Wall Motion}

The discussion will be limited to head-to-head or tailto-tail domain walls in soft magnetic permalloy $\left(\mathrm{Ni}_{80} \mathrm{Fe}_{20}\right)$. Two domain wall types are generally found as shown in Fig. 2. In Fig. 2a, a photoemission electron microscopy (PEEM) [12] image of a vortex head-to-head wall is shown together with a micromagnetic simulation where the spin structure is visualized together with the resulting contrast

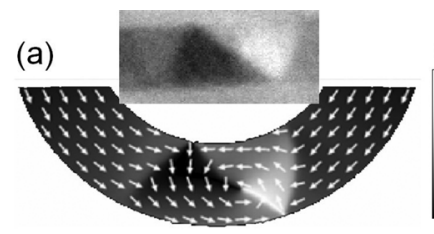

(b)

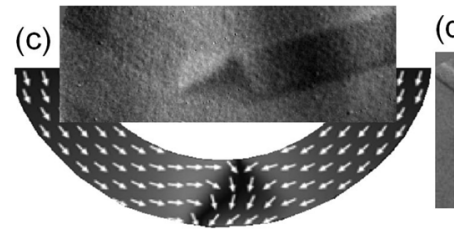

(d)
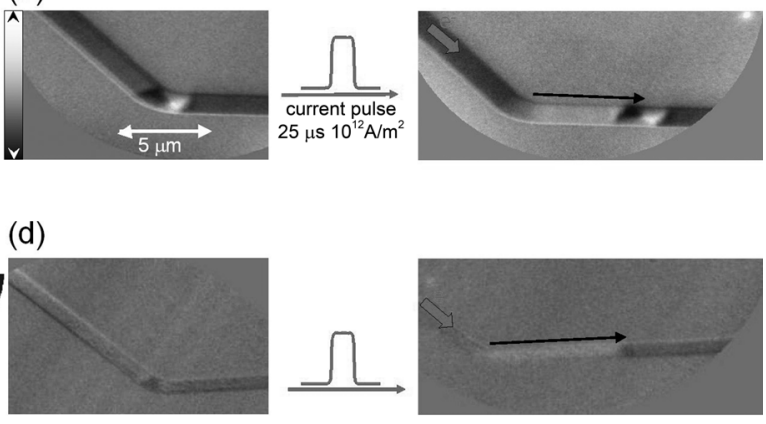

Fig. 2. (colour online): Photoemission electron microscopy images and corresponding micromagnetic simulations of: (a) a vortex head-to-head domain wall; (c) a transverse wall. The shades of grey and the arrows indicate magnetization directions. The image in (b) shows the displacement of a vortex wall by current injection in a $28 \mathrm{~nm}$-thick, 1.0 ìm-wide permalloy wire with an average velocity of approximately $0.3 \mathrm{~m} / \mathrm{s}$. The grey scale bar shows the magnetic contrast direction for all the images. The image in (d) shows the displacement of a transverse wall in a 7.0-nm thick and $500 \mathrm{~nm}$-wide wire. In both cases, the wall spin structure stays the same after the displacement. 
of the image. In Fig. 2c, a transverse wall is shown that occurs in thinner and narrower structures (for details of the geometry-dependence of the wall spin structure see [8]). The influence of a current on a domain wall was investigated theoretically some time ago [13] but has recently seen a revival due to the advent of advanced nanolithography [14] that allows fabrication of well-defined nanoscale magnetic wires. The displacement is due to the transfer (in a simple model) of angular momentum (adiabatic spin torque) and linear momentum (spin torque due to non-adiabatic transport and spin relaxation) from the conduction electrons to the domain wall, thus leading to current-induced domain wall motion in the direction of the electron flow [15-17]. Experimentally, current-induced wall motion has been studied by a large number of groups using various techniques (for an overview, see references in [8, 18-26]). An example of experimentally observed domain wall displacements is shown in Fig. 2b and 2d. The sample is prepared using electron beam lithography, including deposition of permalloy/Au by molecular beam epitaxy on a naturally oxidized Si wafer and a double lift-off process [14]. Here we see in Fig. 2b that a vortex head-to-head domain wall is displaced by a current pulse with a high current density of $10^{12} \mathrm{~A} / \mathrm{m}^{2}$ in the electron flow direction. Dynamic measurements of the velocity have been carried out by Hayashi et al. in the case of a domain wall, which is dynamically generated; high velocities exceeding $100 \mathrm{~m} / \mathrm{s}$ have been reported [26]. Starting with a domain wall at

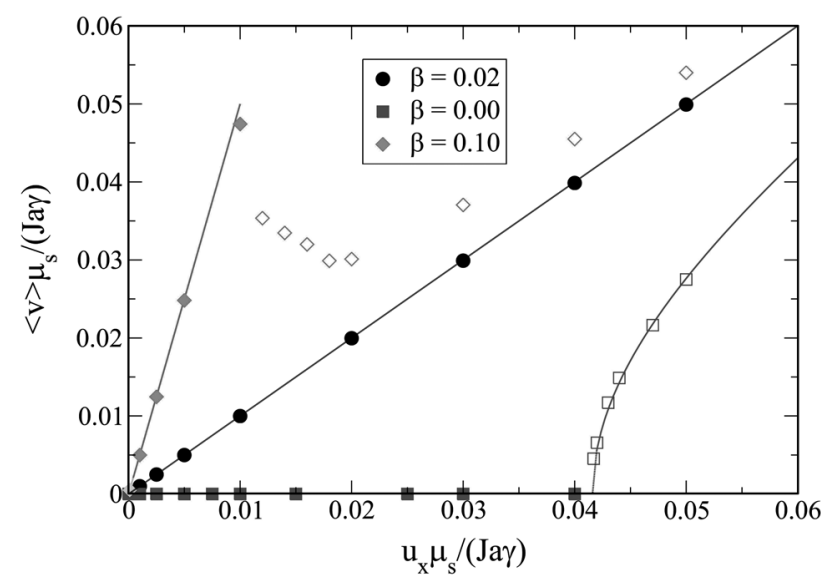

Fig. 3. (from [27]) Simulation of the wall velocity as a function of the injected current density $\mathrm{j}$ (details of the simulation and units used, see [27]). The damping constant used is $\alpha=$ 0.02 , and the 3 different values of the non-adiabaticity parameter $\beta$ are: $\beta=0$ (red empty squares), $\beta=0.02=\alpha$ (black discs), and $\beta=0.1$ (green diamonds). The filled symbols correspond to motion with no domain wall transformation (below the Walker breakdown), while the open symbols signify motion including wall transformations. rest, slower velocities have been observed for pure currentinduced wall motion as seen in Fig. 2 [20,21], and for sufficiently high current densities, periodic wall transformations have been imaged $[21,25]$. The theoretically expected velocity as a function of current density is depicted in Fig. 3 [27]. The behaviour depends on the parameter, which accounts for the torque arising from non-adiabatic transport and spin relaxation that likely dominates for a wide domain wall in permalloy (for details see $[15,16,27])$. For the purely adiabatic case $(\beta=0$, red squares), a high critical current density (jc) is observed as predicted by Thiaville [15] and Zhang [16] and the velocity follows a $\left(j^{2}-j_{c}\right)^{2 / 2}$ behaviour [17] and approaches an effective velocity for large current densities as previously detailed $[8,15]$. If non-adiabatic transport or spin relaxation exist (non-adiabaticity, $\beta \neq 0$ ), the critical current density is reduced to 0 for an ideal wire and the velocity increases at first linearly with current density. For the case that the non-adiabaticity parameter is equal to damping ( $\beta$ $=\alpha$, black discs), the velocity always increases linearly and the wall is not deformed. For $\beta \neq 0$ and $\beta \neq \alpha$ (green diamonds), the velocity scales with $\beta / \alpha$ up to a peak at the Walker current density. Above this, wall transformations occur (open symbols in Fig. 3) [25].

\section{Field-induced Domain Wall Motion}

While current-induced domain wall motion has raised huge interest, a more conventional approach using external magnetic fields has recently received less attention. Nonetheless, there are experimental measurements of depinning fields and velocities [8, 24, 28-32] that shed light on field-driven behaviour. So far, global external fields have been used to displace domain walls, but for a device, the control of a single domain wall is necessary, and for this, localized manipulation of a specific domain wall is sought. To achieve this, localized fields can be used as employed in magnetic random access memory devices [3]. An alternative that was suggested by R. Cowburn [6] is to apply global fields and locally depin a domain wall from a pinning site by manipulating the pinning strength, achieved by local heating [6]. In the following, a more detailed domain wall motion in a permalloy nanowire, influenced by local Oersted fields and Joule heating resulting from a current pulse in an adjacent $\mathrm{Au}$ wire, is addressed. This topic is important due to its potential for developing new technological devices, such as the magnetic shift register based on local thermal activation (schematic, see Fig. 4a-4d) [6, 33, 34]. For the latter, notches are locally heated to reduce the field required to depin a domain wall from a notch. When the external field is 
(a)



(b)

(c)
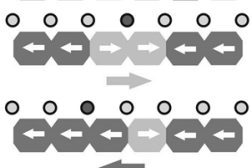

(d)



(e)



Au voltage Au wire contacts

(f)

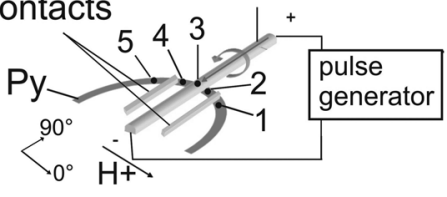

Fig. 4. (colour online) (a)-(d): Schematic of a shift register, in which domains are moved by using thermally-activated domain wall traps. The colours yellow and blue correspond to different magnetization directions. A wire with periodically-arranged notches exhibits different domain configurations between the traps (a). By applying a field, arrow in (b), and heating 1 specific notch (red dot), the domain wall can be selectively depinned at this notch (b). By heating a different notch and applying a field in the opposite direction, arrow in (c), the domain wall is depinned at that notch and is caught at the next notch (c). Thus, the domain is displaced 1 step forward (d), using this slip-stick motion [6]. (e) Optical micrograph of the sample. The bright wire is Py and the dark wires are Au. (f) In this schematic, the relevant sample features are shown. The domain wall is placed in the magnetic Py half ring. Five Au wires, 3 shown in (f), are connected to the Py half ring. The different positions of the domain walls are indicated with numbers 1 to 5 .

applied, only the walls at the heated notches depin and the field then moves the domain wall in the desired direction as explained in Fig. $4 \mathrm{~b}$ and $4 \mathrm{c}$. The sample is fabricated as described in the section of current-induced domain wall motion. The magnetic material permalloy (Py), with a capping layer of $\mathrm{Au}\left(\mathrm{Fe}_{20} \mathrm{Ni}_{80}[40 \mathrm{~nm}] / \mathrm{Au}\right.$ [2.0 nm]) is connected with Au leads of $20 \mathrm{~nm}$ thickness. The sample has no artificial pinning centers due to the notches, but pinning centers due to roughness and other material defects do exist. The structure of interest is a permalloy half ring with a width of $500 \mathrm{~nm}$ and $5 \mathrm{Au}$ wires connected to it (Fig. 4e). The central Au wire, with a cross section of $300 \mathrm{~nm} \times 20 \mathrm{~nm}$, is connected to a pulse generator while the other $4 \mathrm{Au}$ contacts are used for magnetoresistance measurements (Fig. 4f). Due to the dimensions of the permalloy half ring, the presence of vortex domain walls is expected [8]. We use an experimental magnetotransport setup inside a cryostat at a temperature of $4.3 \mathrm{~K}$ to study the wall behaviour. The magnetic field is generated by 2 coil pairs perpendicular to each other so the field can rotate in the plane of the sample. The domain wall position is determined from the anisotropic magnetoresistance since it depends on whether there is a domain wall between the Au wires (low resistance) or not (high resistance) [35]. To demonstrate this, the domain wall was positioned between the voltage contacts and depinned as shown in Fig. 5. In this figure, the depinning at $29 \mathrm{G}$ is detected due to the accompanying increase in the resistance when the domain wall leaves the area between the contacts where the resistance is measured. To selectively position the domain wall between the voltage contacts ( $\mathrm{Au}$ wires next to the central Au wire), a field of $5000 \mathrm{G}$ in a specific direction was applied, followed by

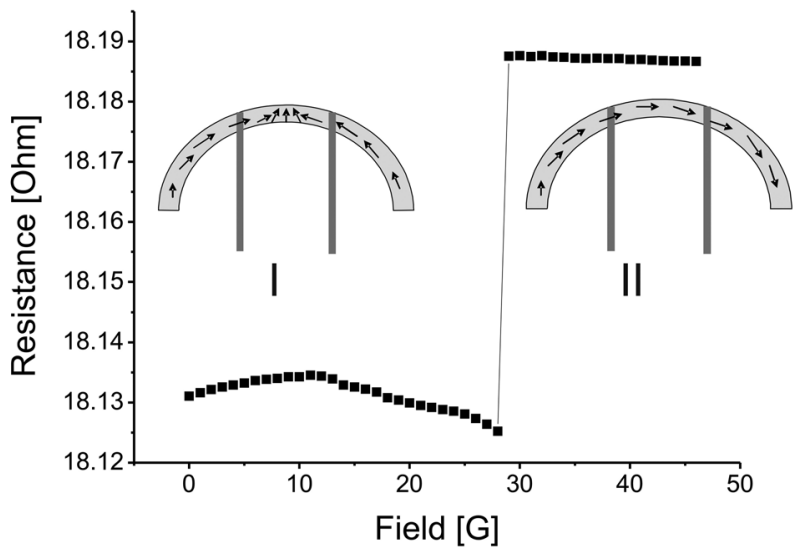

Fig. 5. (colour online) Change in resistance as a domain wall is moved out of the area between the voltage contacts. The low resistance at 0 field corresponds to the domain wall being present between the contacts as shown in the schematic. As the field is increased, the domain wall is depinned at $29 \mathrm{G}$ and this is reflected in the jump of the resistance to a higher level. The resistance change is $0.3 \%$ and is easily detectable using a lockin technique.

reduction of the field to 0 and measurement of the resistance. The different resistance levels for the different domain wall positions are shown in Fig. 6a. Here the sample was saturated along different directions and then, after relaxing the field back to 0 , the domain wall is located at the position corresponding to the field direction. Four different resistance levels are visible for the different wall positions. The highest levels correspond to the resistance when the domain wall is outside the region between the voltage contacts $\left(<73^{\circ}\right.$ and $\left.>104^{\circ}\right)$, positions 1 and 5 in Fig. 4f. The resistance level is lower when the domain wall is located at position 2 between the voltage contacts $\left(74^{\circ}-85^{\circ}\right)$. The central level 3 shows a small 
increase in the resistance in the range of $86^{\circ}-93^{\circ}$, when the domain wall is located at the $\mathrm{Au} / \mathrm{Py}$ interface below the central Au wire that shunts part of the current for the MR measurement (position 3). In the range of $94^{\circ}$ to $102^{\circ}$, the domain wall is held at a pinning center partly below the Au/Py interface (position 4), giving a higher resistance than at position 2. In the next step, the field for the depinning of the domain wall from the pinning site at position 4 is studied as a function of temperature. The necessary field depends on the angle of the applied field and is minimal if the field points perpendicularly to the central Au wire (along $0^{\circ}$ or $180^{\circ}$ ) [36]. Pinning centers due to edge roughness or other defects increase the depinning field of the domain wall [28]. To move the domain wall, the depinning field $\mathrm{H}_{\mathrm{d}}$ that needs to be applied has a dependence on the temperature that can be described using the following model $[37,38]$ :

$$
H_{d}(T) \cong H_{0}\left\{1-\left[\frac{k_{b} T}{E_{0}} \cdot \ln \left(\frac{\Gamma_{0} k_{b} T H_{0}}{1.5 E_{0} v\left(1-\frac{H_{d}}{H_{0}}\right)^{1 / 2}}\right)\right]^{2 / 3}\right\},
$$

The parameters are the field sweep rate $(v)$, the attempt frequency at zero temperature $\left(\Gamma_{0}\right)$, the depinning field at 0 temperature $\left(H_{0}\right)$, and the energy barrier $\left(E_{0}\right)$ for no applied field. This equation was derived by Lok et al. and stems from the Kurkijärvi model [37]. To depin the domain wall, an external in-plane field perpendicular to the central Au wire was applied. In Fig. 6b, the results for the depinning field at different cryostat temperatures are presented. The lowering of the depinning field due to thermal activation is visible. To obtain the energy barrier $E_{0}$ from Eq. (1), the following parameters are used:

$$
\Gamma_{0}=10^{9} \mathrm{~Hz}, v=2 \mathrm{Oe} / \mathrm{s} \text { [38] and } H_{d} / H_{0}=0.58 \text {. }
$$

This ratio is the average of the value of $H_{d} / H_{0}$ at 4.3 and $298 \mathrm{~K}$, although the result for $E_{0}$ is not very sensitive to the $H_{d} / H_{0}$ ratio. For a positive field, we find $E_{0}=1.37$ $\times 10^{19} \mathrm{~J}$, and for a negative field, $E_{0}=1.19 \times 10^{19} \mathrm{~J}$ is in line with earlier experiments $[38,39]$. Thus, from this we find that heating indeed reduces the depinning field. To generate this heating locally at the domain wall, a current is passed through the Au wire and the heating due to a current pulse is determined. To achieve this, the resistance of the Au wire is first measured as a function of temperature in the cryostat. The resistance of the Au wire is then determined during the current pulse injection as a function of current density and from this, the heating of the $\mathrm{Au}$ wire is determined as a function of current density $[40,41]$. In the following, a current pulse with a length of $50 \mu \mathrm{s}$ and a rise time of $18 \mu \mathrm{s}$ is used. As seen in Fig. 6c, the highest current density temperatures are obtained by heating to more than $100 \mathrm{~K}$. To ascertain the effect of the Oersted field on the domain wall, the Oersted field was calculated numerically, assuming a uniform current distribution in the Au wire. The Oersted field was found to be inhomogeneous and while it points in plane at the centre of the wire, there are strong out-of-plane components at the edges of the wire. At $6.6 \times 1011 \mathrm{~A} / \mathrm{m}^{2}$, an Oersted field at the interface of the Au wire with the permalloy wire of up to 80 Oe is expected, which decays with

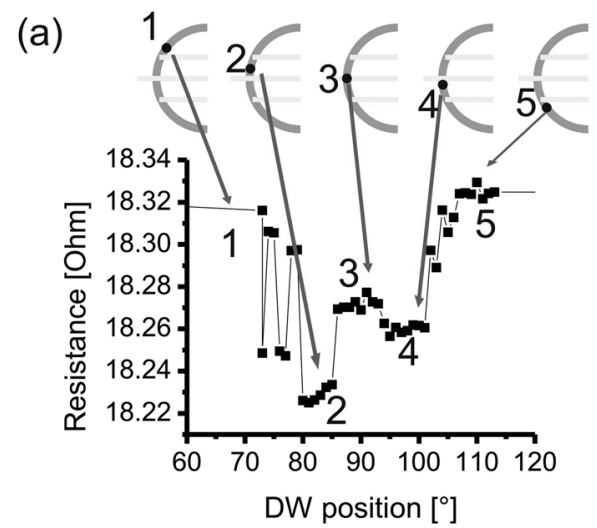

(b)
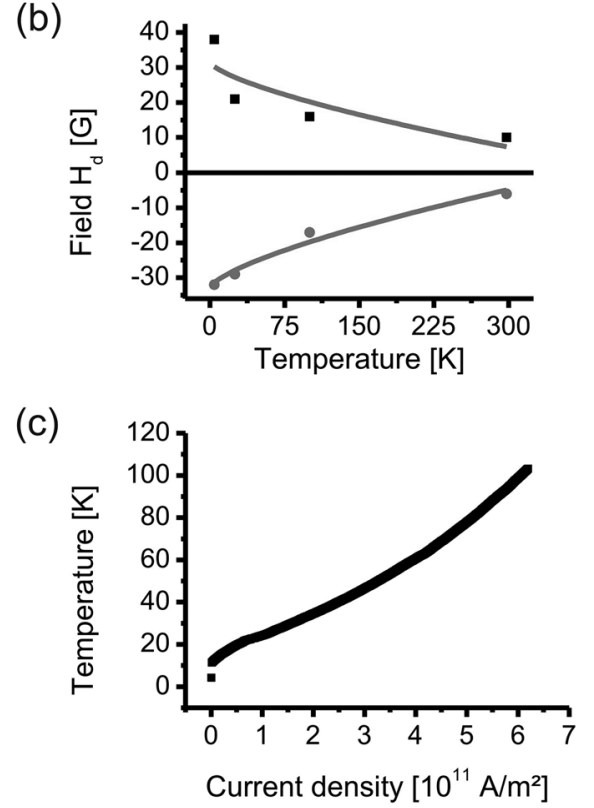

Fig. 6. (colour online) (a) Magnetoresistance measurement at $4.3 \mathrm{~K}$ as a function of the angle of the saturation field (as detailed in [35]). The domain wall positions are labeled by numbers, which correspond to those in Fig. 4. The corresponding domain wall positions are indicated in the schematics above. (b) Temperature dependence of depinning fields for a positive field (black squares) and a negative field (red dots) for the domain wall placed at $98^{\circ}$. The red line is a fit curve using the model of Kurkijrvi [37]. (c) Temperature of the Au wire as a function of the injected current density determined from measuring the resistance during pulse injection. 
(a) Head-to-head Domain Wall
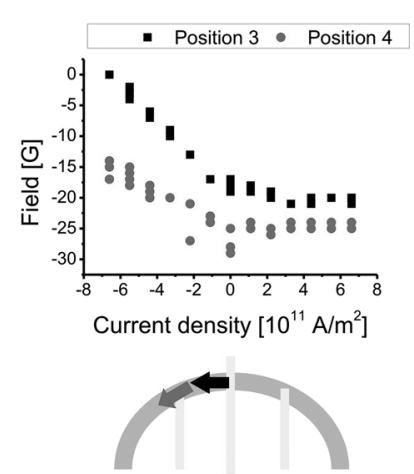

schematic I

(e) Head-to-head Domain Wall

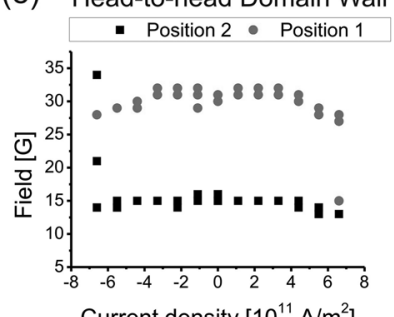

Current density $\left[10^{11} \mathrm{~A} / \mathrm{m}^{2}\right]$
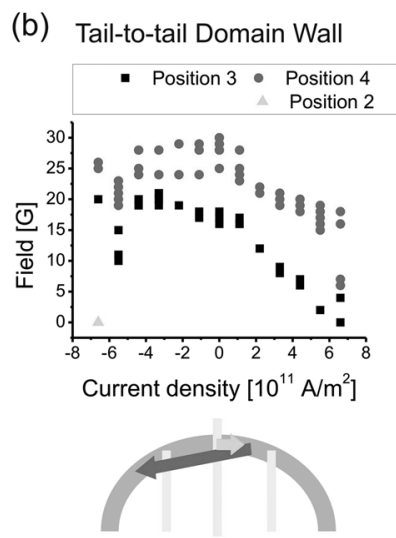

schematic II

(f) Tail-to-tail Domain Wall

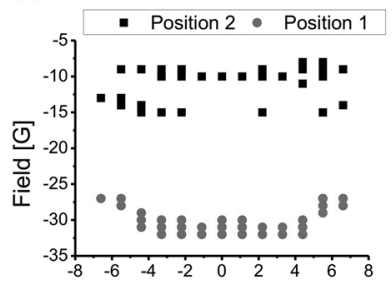

Current density $\left[10^{11} \mathrm{~A} / \mathrm{m}^{2}\right]$

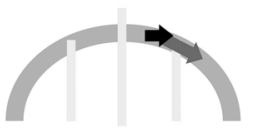

schematic $\mathrm{V}$ (c) Head-to-head Domain Wall
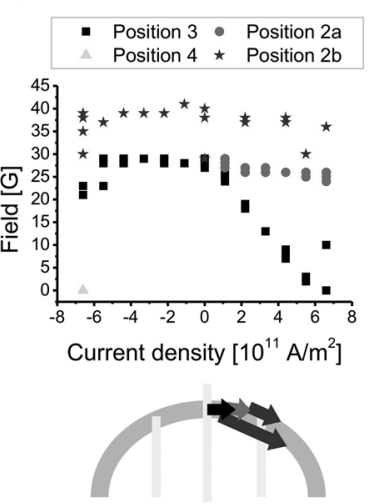

schematic III (d) Tail-to-tail Domain Wall

- Position 3 - Position 2a



Current density $\left[10^{11} \mathrm{~A} / \mathrm{m}^{2}\right]$

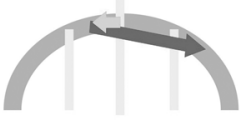

schematic IV

(g) Head-to-head Domain Wall

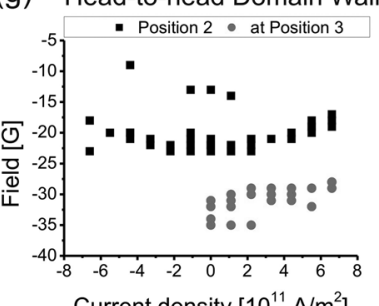

Current density $\left[10^{11} \mathrm{~A} / \mathrm{m}^{2}\right]$



schematic $\mathrm{VI}$

Fig. 7. (colour online) Domain wall propagation fields for different applied field and current pulses for domain walls positioned originally at $90^{\circ}$, position $3,(\mathrm{a}),(\mathrm{b})$, (c), and (d), and $82^{\circ}$, position $2,(\mathrm{e}),(\mathrm{f})$, and (g). The black squares and green up triangles indicate the first displacement from the original position and the red discs and blue stars the displacement outside the area between the contacts (to position 1 or 5). The depinning fields are shown as a function of current density for a domain wall starting at position 3: (a) negative external fields and a head-to-head wall; (b) positive external fields and a tail-to-tail wall; (c) positive fields and a head-to-head wall; (d) negative fields and a tail-to-tail wall. For a domain wall starting at position 2: (e) positive external fields and a head-to-head wall; (f) negative external fields and a tail-to-tail wall; (g) negative external fields and a head-to-head wall. The schematics below the graphs visualize the domain wall motion direction in the sample and the colours of the arrows correspond to the colours of the symbols in the graphs.

increasing distance from the interface. The Oersted field decreased to approximately 15 Oe at position 2 and is close to 0 Oe at positions 1 and 5 . For position 4 , the Oersted field was near $60 \mathrm{Oe}$, if a DW within a distance of $50 \mathrm{~nm}$ from the Au wire is assumed. The change of the depinning field of a domain wall for different current pulses through the central Au wire with a current density up to $6.6 \times 1011 \mathrm{~A} / \mathrm{m}^{2}$ at $4.3 \mathrm{~K}$ was studied experimentally. Here, no current was injected into the Py. The primary focus lies upon a domain wall position below the central $\mathrm{Au}$ wire placed by a field in the direction of $90^{\circ}$ (position 3, Fig. 4f), and on an adjacent position $150 \mathrm{~nm}$ away from the central wire, placed by a field in a direction of $82^{\circ}$ (position 2, Fig. 4f). The data points in Fig. 7 correspond to the first domain wall motion away from its original position, and then the domain wall motion out of the area between the voltage contacts.

First, a head-to-head domain wall was positioned underneath the central $\mathrm{Au}$ wire (position 3) by applying an external field at $90^{\circ}$ and relaxing the field along this direction back to 0 . Fig. 7a shows the effect of a negative applied depinning field along $0^{\circ}$ that tries to move the head-to-head domain wall towards position 5 and the effect of current pulses with different polarities that cause an Oersted field of different signs. A positive current density corresponds to an Oersted field antiparallel to the external field and thus tries to move the wall towards position 1. A negative current density has an Oersted field parallel to the external field and thus acts in the same direction, moving the wall towards position 5. For no 
current pulse ( 0 current density), there are 2 main movements of the domain wall: the first displacement is from position 3 (Py/Au-interface) at about $-18 \mathrm{G}$ to a pinning site at position 4 (black squares); the second to the area outside the contacts, at about $-25 \mathrm{G}$ (red discs). These 2 displacements are visualized in schematic I, below the graph (Fig. 7a). On applying negative current pulses, the depinning field for the first domain wall movement (position 3 to 4 ) decreases with increasing current density since the Oersted field of the current pulse through the central $\mathrm{Au}$ wire supports the wall motion given the external field. At a maximum current density of $-6.6 \times 10^{11} \mathrm{~A} / \mathrm{m}^{2}$, the wall moves purely due to the Oersted field with no externally applied field. The second domain wall displacement field (red discs) decreases slightly because the Oersted field extends to position 4 , which is a pinning center next to the central Au wire. For positive current pulses, the depinning field for the first motion is only slightly reduced due to Joule heating. The Oersted field hinders the fieldinduced domain wall motion and the domain wall moves only due to the applied field. The same behavior is observed for a tail-to-tail wall (Fig. 7b), but not as expected for reversed field and current directions. In addition, for a very large negative current density $\left(-6.6 \times 10^{11} \mathrm{~A} /\right.$ $\mathrm{m}^{2}$ ) the wall moves purely due to the Oersted field at a 0 applied external field (green triangle). In this case, the wall first moves to position 2, which is in the opposite direction to the external field, and then due to the external field to position 5 as shown in Fig. 7b, schematic II. A positive field is then applied to the head-to-head domain wall (Fig. 7c), causing the head-to-head domain wall to move from position 3 to position 2 and then to position 1 (area outside the voltage contacts, see Fig. 7 schematic III). At 0 current, the first motion from position 3 is at about $27 \mathrm{G}$ to position 2 (black squares). The motion to the area outside the contacts occurs at approximately 38 $\mathrm{G}$ (red discs, blue stars). We find for the first domain wall, analogous movement behaviour to the negative field case shown in Fig. 7a. For a positive current density, where the Oersted field acts in the same way as the external field, the depinning field is reduced with increasing current density until at the highest current density, the wall moves purely due to the Oersted field at 0 applied external field. However, the second movement exhibits a slightly different behaviour for a positive current density. If one looks carefully, the second movement actually consists of 2 displacements and the 2 positions, 2a (red discs) and $2 \mathrm{~b}$ (blue stars), can be distinguished by slightly different resistance levels. For the jumps away from positions $2 \mathrm{a}$ and $2 \mathrm{~b}$, there is only a small decrease in the wall displacement field with increasing current (and thus
Oersted field), in contrast to Fig. 7a (red discs), indicating that in the first motion, the domain wall has moved far enough away from the Au wire so that the Oersted field does not significantly contribute to the second displacement in contrast to the cases in Fig. 7a. For negative current densities, there is no significant effect, thus the Joule heating, which would be independent of the current pulse direction, is less important. Thus, the domain wall starts moving when the applied field is high enough, here at a value similar to that at 0 current pulse. For very high current densities, the Oersted field moves the domain wall in the direction opposite to the applied field, see Fig. 7 schematic IV. For a tail-to-tail wall, analogous behaviour is observed as seen when comparing Fig. $7 \mathrm{~d}$ with the data from Fig. 7c. This shows that, as expected, there is no difference between a head-to-head and a tail-to-tail wall. Next, a head-to-head domain wall was placed $150 \mathrm{~nm}$ away from the central wire at $82^{\circ}$ and the depinning behaviour when external fields were applied and currents injected through the central Au wire were observed. The positive applied fields (Fig. 7e) that move the domain wall away from the central wire, towards position 1 (see schematic V), and to a pinning center near the voltage contact are to be considered. Here, a decreasing depinning field symmetric at approximately 0 current was found, indicating pure Joule heating with no effect of the Oersted field, since this should be asymmetric with respect to the current polarity. This is because the domain wall is far enough away from the central Au wire so that there is no significant Oersted field at this position. The same behaviour is found again for a tail-to-tail wall as shown in Fig. 7f. The depinning field ranges between 15 and $13 \mathrm{G}$ for the first motion (black squares) and between 30 and $27 \mathrm{G}$ for the second motion (red discs). Using Eq. (1), this change arises from a temperature rise of $24 \mathrm{~K}$ and $15 \mathrm{~K}$. To understand the heating, this is compared to the heating in the $\mathrm{Au}$ wire determined for the maximum current density, which was $110 \mathrm{~K}$ (Fig. 6c). This means that the temperature drops by about $90 \mathrm{~K}$ within $150 \mathrm{~nm}$, so that Joule heating can also be treated as a rather localized effect. Finally, the behaviour of the head-to-head domain wall positioned at $82^{\circ}$ when a negative external field is applied was observed. In Fig. $7 \mathrm{~g}$, the fields for the domain wall motion as a function of current density are noticeable and a very asymmetric behaviour with current polarity arises. For 0 and positive current densities, 2 domain wall motion jumps (black squares and red discs) become apparent, while for negative current densities, only a single displacement was observed. For the 0 current case, the first displacement occurred near $-22 \mathrm{G}$ from the original position towards the central $\mathrm{Au}$ wire. 
The second displacement occurred at $-31 \mathrm{G}$ and moved the domain wall out of the area between the contacts towards position 5. This also holds for positive current densities where the Oersted field at the central Au wire hinders the domain wall from going across the Au wire towards position 5. For negative current densities, the Oersted field supports the motion of the head-to-head wall towards position 5 , so that the domain wall is not pinned close to the central $\mathrm{Au}$ wire but directly moved at the depinning field of $-22 \mathrm{G}$ to position 5 . This occurred even for small current densities that generated only a few G, showing that even a small field can suffice to support the wall motion, once the wall is moving. This dynamic wall propagation field that is much smaller than the quasistatic depinning field was also recently observed and measured by Beach et al. [30] and Thomas et al. [42].

\section{Alternative Approaches To Domain Wall Motion}

In addition to these 2 approaches presented above, further schemes have been devised to induce wall motion. Inhomogeneous magnetic fields transverse to the wire have been proposed [43], where the energy of the transverse component of the magnetization in the wall changes with the position. This is due to the inhomogeneity of the field so that the walls are pulled towards a position where the field lowers their energy. However, due to the need for the generation of inhomogeneous fields, the experimental realization might be rather challenging. Another possibility is to employ pure diffusive spin currents. In this case, a non-local spin valve geometry is used [44-47] to generate a pure spin current. When this spin current is absorbed by a ferromagnet, a change in the magnetization of the ferromagnet is possible, as seen in the reversal of a small dot $[45,48]$. Domain wall nucleation has also been assisted by spin currents [49] and first results of wall displacement due to spin current absorption have been presented [50]. While this approach is very exciting from a fundamental physics perspective, several problems may be encountered if a large-scale realization is sought. Generating spin currents is not straightforward, and the magnitude of the spin accumulation due to the spin current is mostly small due to the limited spin injection efficiency and the finite diffusion length (even though novel materials [51] might provide longer spin diffusion lengths). Finally, magnetization reversal by optical techniques has been recently demonstrated [52] and it might be possible to displace domains and domain walls by carefully designing excitations of the magnetization with a laser.

\section{Conclusions}

In conclusion, the different concepts for domain wall motion based on currents and fields have been reviewed. Currents allow for vortex and transverse wall displacement due to the spin transfer torque effect and in this case, all walls (head-to-head and tail-to-tail) move in the direction of the electron flow. In the case of field-induced motion, a more complex concept needs to be developed, since headto-head and tail-to-tail walls move in opposite directions when a uniform field is applied. By local heating of pinning sites, field-induced slip-stick wall motion can be achieved that allows selective movement of domains and domain walls by varying pinning strength. The motion of domain walls in a permalloy half-ring nanostructure with an $\mathrm{Au}$ wire across it has also been investigated. The current through the $\mathrm{Au}$ wire generates an Oersted field and heats the permalloy locally at the interface between the Au and permalloy. The finding that the Oersted field has a short range indicates that through Au wires, the wall motion behavior can be locally tailored by injecting appropriate current pulse heights and polarities to generate the necessary Oe field pulses. The dynamic propagation field required for domain wall motion, once a wall is depinned, is much smaller than the quasi-static depinning field. Due to the short range of the Joule heating and Oersted field effect, domain walls positioned more than $150 \mathrm{~nm}$ away are not significantly affected, so that short distances between walls and thus high storage densities can be achieved. Furthermore, by appropriately designing artificial pinning sites at short enough distances [39], walls from one pinning site can be moved to the next just by using the Oersted field with no externally applied field. This opens up a new avenue for simple device operation, such as pinning with notches in a ferromagnetic wire and conducting non-magnetic wires at the position of the notches that generate controlled local fields to implement a full device.

The authors acknowledge support by the DFG (KL1811, SFB 513, and SFB 767), the E.U. (Human Resources and Mobility Programme and SPINSWITCH MRTN-CT-2006035327), the European Research Council, through a Starting Independent Researcher Grant (ERC-2007-StG 208162), the Zukunfskolleg of the University of Konstanz and the Samsung Advanced Institute of Technology.

\section{References}

[1] I. R. McFadyen, E. E. Fullerton, and M. J. Carey, MRS Bull. 31, 379 (2006).

[2] R. H. Dee, MRS Bull. 31, 404 (2006). 
[3] S. Tehrani, J. M. Slaughter, E. Chen, M. Durlam, J. Shi, and M. DeHerrera, IEEE Trans. Mag. 35, 2814 (1999).

[4] S. S. P. Parkin, U.S. patent $6,834,005$ and patent application 10/984,055 (2004).

[5] S. S. P. Parkin, M. Hayashi, and L. Thomas, Science 320, 190 (2008).

[6] R. Cowburn, D. Petit, D. Read, and O. Petracic, Patent WO 2007/132174A1.

[7] S. S. P. Parkin, MRS Bull. 31, 389 (2006).

[8] M. Kläui, J. Phys. Condens. Matter 20, 313001 (2008).

[9] J. A. C. Bland, M. Kläui, L. Lopez-Diaz, and J. Rothman, Patent WO 0213208.

[10] D. A. Allwood, G. Xiong, M. D. Cooke, C. C. Faulkner, D. Atkinson, N. Vernier, and R. P. Cowburn, Science 296, 2003 (2002).

[11] D. A. Allwood, G. Xiong, C. C. Faulkner, D. Atkinson, D. Petit, and R. P. Cowburn, Science 309, 1688 (2005).

[12] J. Stöhr, Y. Wu, B. D. Hermsmeier, M. G. Samant, G. R. Harp, S. Koranda, D. Dunham, and B. P. Tonner, Science 259, 658 (1993).

[13] L. Berger, J. Appl. Phys. 55, 1954 (1984).

[14] L. J. Heyderman, M. Kläui, B. Nöhammer, C. A. F. Vaz, J. A. C. Bland, and C. David, Microelec. Eng. 73-74, 780 (2004).

[15] A. Thiaville, Y. Nakatani, J. Miltat, and Y. Suzuki Europhys. Lett. 6, 990 (2005).

[16] S. Zhang and Z. Li, Phys. Rev. Lett. 93, 127204 (2004).

[17] G. Tatara and H. Kohno, Phys. Rev. Lett. 92, 86601 (2004).

[18] L. Thomas, M. Hayashi, X. Jiang, R. Moriya, C. Rettner, and S. S. P. Parkin, Nature 443, 197 (2006).

[19] G. Meier, M. Bolte, R. Eiselt, B. Krüger, D.-H. Kim, and P. Fischer, Phys. Rev. Lett. 98, 17187202 (2007).

[20] A. Yamaguchi, T. Ono, S. Nasu, K. Miyake, K. Mibu, and T. Shinjo, Phys. Rev. Lett. 92, 077205 (2004).

[21] M. Kläui, C. A. F. Vaz, J. A. C. Bland, W. Wernsdorfer, G. Faini, E. Cambril, L. J. Heyderman, F. Nolting, and U. Rüdiger, Phys. Rev. Lett. 94, 106601 (2005); ibid 95, 26601 (2005).

[22] J. Grollier, P. Boulenc, V. Cros, A. Hamzic, A. Vaurs, and A. Fert, Appl. Phys. Lett. 83, 509 (2003).

[23] C. H. Marrows, Adv. Phys. 54, 585 (2005).

[24] M. Hayashi, L. Thomas, C. Rettner, R. Moriya, and S. S. P. Parkin, Nature Phys. 3, 21 (2007).

[25] L. Heyne, M. Kläui, D. Backes, T. A. Moore, S. Krzyk, U. Rüdiger, L. J. Heyderman, A. Fraile Rodriguez, F. Nolting, T. O. Mentes, M. A. Nino, A. Locatelli, K. Kirsch, and R. Mattheis, Phys. Rev. Lett. 100, 66603 (2008).

[26] M. Hayashi, L. Thomas, C. Rettner, R. Moriya, Y. B. Bazaliy, and S. S. P. Parkin, Phys. Rev. Lett. 98, 37204 (2007).

[27] C. Schieback, M. Kläui, U. Nowak, U. Rüdiger, and P. Nielaba, Eur. Phys. J. B 59, 429 (2007).

[28] M. Kläui, H. Ehrke, U. Rüdiger, T. Kasama, R. E. DuninBorkowski, D. Backes, L. J. Heyderman, C. A. F. Vaz, J. A. C. Bland, G. Faini, E. Cambril, and W. Wernsdorfer, Appl. Phys. Lett. 87, 102509 (2005).
[29] D. Atkinson, D. A. Allwood, G. Xiong, M. D. Cooke, C. C. Faulkner, and R. P. Cowburn, Nature Materials 2, 85 (2003).

[30] G. S. D. Beach, C. Nistor, C. Knutson, M. Tsoi, and J. L. Erskine, Nature Mater. 4, 741 (2005).

[31] M. Hara, T. Kimura, and Y. Otani, Appl. Phys. Lett. 90, 242504 (2007).

[32] D. Petit, A.-V. Jausovec, D. Read, and R. P. Cowburn, J. Appl. Phys. 103, 114307 (2008).

[33] D. Ilgaz, M. Kläui, L. Heyne, O. Boulle, F. Zinser, S. Krzyk, M. Fonin, U. Rüdiger, D. Backes, and L. J. Heyderman, Appl. Phys. Lett. 93, 132503 (2008).

[34] S.-S. Ha and C.-Y. You, J. Magn. 12, 7 (2007).

[35] M. Kläui, C. A. F. Vaz, J. Rothman, J. A. C. Bland, W. Wernsdorfer, G. Faini, and E. Cambril, Phys. Rev. Lett. 90, 097202 (2003).

[36] D. Bedau, M. Kläui, U. Rüdiger, C. A. F. Vaz, J. A. C. Bland, G. Faini, L. Vila, and W. Wernsdorfer, J. Appl. Phys. 101, 09F509 (2007).

[37] J. G. S. Lok, A. K. Geim, U. Wyder, J. C. Manan, and S. V. Dubonos, J. Magn. Magn. Mater. 204, 159 (1999).

[38] A. Himeno, T. Okuno, T. Ono, K. Mibu, S. Nasu, and T. Shinjo, J. Magn. Magn. Mater. 286, 167 (2005).

[39] P. Dagras, M. Kläui, M. Laufenberg, D. Bedau, L. Vila, G. Faini, C. A. F. Vaz, J. A. C. Bland, and U. Rüdiger, J. Phys. D. Appl. Phys. 40, 1247 (2007).

[40] A. Yamaguchi, S. Nasu, H. Tanigawa, T. Ono, K. Miyake, K. Mibu, and T. Shinjo, Appl. Phys. Lett. 86, 012511 (2005).

[41] M. Laufenberg, W. Bührer, D. Bedau, P.-E. Melchy, M. Kläui, L. Vila, G. Faini, C. A. F. Vaz, J. A. C. Bland, and U. Rüdiger, Phys. Rev. Lett. 97, 046602 (2006).

[42] L. Thomas, Presentation 7036-33, SPIE Spintronics conference (2008).

[43] C.-Y. You, Appl. Phys. Lett. 92, 152507, (2008); ibid 192514, (2008).

[44] M. Johnson and R. H. Silsbee, Phys. Rev. Lett. 55, 1790 (1985).

[45] T. Kimura, Y. Otani, and J. Hamrle, Phys. Rev. Lett. 96, 37201 (2006).

[46] F. J. Jedema, A. T. Filip, and B. van Wees, Nature 410, 345 (2001).

[47] Y. Ji, A. Hoffmann, J. S. Jiang, J. E. Pearson, and S. D. Bader, J. Phys. D: Appl. Phys. 40, 1280 (2007).

[48] T. Yang, T. Kimura, J.-B. Laloe, and Y. Otani, Nature Phys. 4, 851 (2008).

[49] T. Kimura and Y. Otani, J. Phys. D: Appl. Phys. 40, 1285 (2007)

[50] M. Kläui, Presentation at the Yukawa Workshop Spin Transport in Condensed Matter, Kyoto (2008); (unpublished).

[51] N. Tombros, C. Jozsa, M. Popinciuc, H. T. Jonkman, and B. van Wees, Nature 448, 571 (2007).

[52] A. V. Kimel, A. Kirilyuk, P. A. Usachev, R. V. Pisarev, A. M. Balbashow, and T. Rasing, Nature 435, 655 (2005). 